\title{
DDEMA: A Data Driven Environment for Multiphysics Applications
}

\author{
John Michopoulos ${ }^{1}$, Panagiota Tsompanopoulou ${ }^{2}$, Elias Houstis ${ }^{3,2}$, \\ John Rice ${ }^{3}$, Charbel Farhat ${ }^{4}$, Michel Lesoinne ${ }^{4}$, and Frederic Lechenault ${ }^{4}$ \\ 1 U.S. Naval Research Laboratory, Special Projects Group, Code 6303 \\ Washington, DC 20375, U.S.A. \\ john.michopoulos@nrl.navy.mil \\ 2 University of Thessaly, Dept. of Comp. Eng. and Telecommunications \\ 38221 Volos, Greece \\ yota@inf.uth.gr \\ 3 Purdue University, Computer Sciences Department \\ W. Lafayette, IN 47906, U.S.A. \\ $\{$ enh, jrr\}@cs.purdue.edu \\ 4 University of Colorado at Boulder, Dept. of Aerospace Engineering Sciences \\ Boulder, CO 80309-0429, U.S.A. \\ \{farhat, michel, lechenault\}@colorado.edu
}

\begin{abstract}
In this paper we present the design of a multidisciplined problem solving environment (MPSE) for supporting an efficient prediction capability for the response of multiscale interdisciplinary continuous interacting systems. This design takes into consideration information technologies, coupled multiphysics sciences, and data-driveness to steer adaptive modelling and simulation of the underlying systemic behavior. The paper describes the design objectives and software architecture of DDEMA in the context of two multidisciplinary applications related to material/structure design of supersonic platforms and fire/material/environment interaction monitoring, assessment and management.
\end{abstract}

\section{Introduction}

Continuous interacting systems under the influence of coupled multifield loading can exhibit static and dynamic behavior that is highly variable in space and time, spans multiple scales, and sometimes has intense nonlinear interaction and response. This is true especially for material/structural systems embedded in host continua as in the case of structures interacting with surrounding fluids under generalized loading conditions. The development of the multiphysics science and technology that allows predicting behavioral characteristics of such interacting systems is of utmost importance when it comes to their applications in contexts driven by human needs. Examples of such applications are: a) Energy storing and dissipating composite materials for supersonic and hypersonic multi-mission conventional or uninhabited air vehicles for civil and defense applications. b) Materials and structures for the civil and defense infrastructure life extension and 
maintainability, such as smart materials and systems that can self-adjust when a scheduled or unexpected restriction of their state space occurs. An example would be high temperature structural composites that can change their mechanical properties when exposed to sudden temperature gradients due to explosion or mechanical damage.

Specific vertical industries must comply with consumer driven quality standards and/or demands that introduce implicit requirements for reducing uncertainty of simulated behavior. This has increased the crucial aspect of the validation and verification of predictions, rendered by the computational environments used for this purpose, up to the point of becoming one of the main areas if intensive work. The main objective of our project is to develop a framework for applying specific Multidisciplinary Problem Solving Environments (MPSEs) to multidisciplinary applications. An instantiation of this framework will be a particular MPSE, which will provide simulation based behavior prediction of fully identified systems as a consequence of applying inverse approaches along with massive automated data acquisition during tests of continuous systems where the tests are exhaustive of multidimensional stimulus-response spaces. Such a data-driven framework will allow the experimental capturing of the multiphysics behavioral characteristics of the continuous system, thus intrinsically ensuring the verifiability and validity of simulations based on the testing. To develop and validate, within the framework provided by this objective, the proposed data driven distributed MPSE will employ appropriate methodologies that will demonstrate two main features. The first feature is a software encapsulation of multiphysics data-driven constitutive characterization and model selection and encapsulation. The second feature is heavy- and light-weight computational solution implementations and comparison for solving of multi domain coupling (i.e. aero-structural interaction) problems. We will be referring to this data-driven MPSE as a Data Driven Environment for Multiphysics Applications (DDEMA) thereafter.

\section{MPSE Overview and DDEMA Specifications}

Progress towards the solution of coupled multiphysics and multi-continua interaction both from the constitutive and the field equation perspectives has been scarce. There is significant progress however, when focus has been given to computational implementations for a small number (mostly three) of interacting fields and a number of active projects exist. Among them one can include our development of the GasTurbLab [1], [2] multidisciplinary problem solving environment (MPSE) that addresses the interactions between a stator, rotor, and combustor in a gas turbine engine that has been implemented on a mobile agent platform, utilizing a collaborating partial differential equations (PDEs) methodology [3], [4]. In the area of fluid-structure-control interaction, we can mention the advanced AERO computational platform. This platform comprises the AERO-F, AERO-S, AERO-C and MATCHER suite of codes, [5],[6], developed at the University of Colorado for the solution on nonlinear transient aeroelastic problems. They are portable, and run on a large variety of comput- 
ing platforms ranging from Unix workstations to shared as well as distributed memory massively parallel computers. A generalization of AERO to an aerothermoelastic implementation was recently achieved [7]. The case of aerostructural coupling with an energy dissipative non-linear material response under a virtual wind tunnel environment has also been initiated recently [8], [9]. The DDEMA system will provide high confidence prediction capability through its data-driven system modeling capability.

The DDEMA system will use network based distributed computing infrastructures and data and will provide a web-accessible user's environment. The envisioned computing infrastructure will have the following properties: (1) Computation will be partitioned into coarse-grain and fine-grain components and will be executed in a loosely coupled fashion for the lightweight implementation, and in tightly coupled fashion for the heavyweight implementation. (2) The system will distribute dynamically the various components in order to adapt according to resource availability and performance variations. (3) To enable automatic adaptive modelling, the system will be minimizing the difference between stored behavioral datasets and predicted behavioral datasets, in a design optimization fashion, where the objective function and the potential constraints will be user or system definable. (4) To enable optimal user customization and history maintenance of user-system interaction, a visual problem definition editor (VPDE) will be developed and its user-invoked states will be archiveable and unarchiveable (or serializable and deserializable) upon request. (5) To enable collective and dynamic knowledge transfer among distributed points of entry and achieve auto enhancement of simulation due to historical collective user experience, abstractions of successful simulations and/or problem solutions as well as their least upper bound (as a representation of the non conflicting historic common asserted knowledge) will be maintained for all users with appropriate authorization/authentication. (6) The system will deal with machine and programming language heterogeneity problems while it will preserve platform independent component communication by leveraging efficient and ubiquitous data exchange formats and methodologies.

The proposed work will borrow design concepts from or build on top of: (1) existing agent systems (e.g. [10]), (2) communications tools that achieve interoperability between different platforms (e.g. Java-RMI-IIOP [11]), (3) symbolic algebra systems and sub systems for automating the field equation generation process (e.g. MathTensor [12]), (4) flexible APIs for visual editing of user controlled problem definition as well as $2 \mathrm{D}$ and $3 \mathrm{D}$ visualization (e.g. PTOLEMY-II [13]), (5) high performance Java implementations for parallel computation over distributed and shared memory architectures focused on intra-paradigm compatibility (e.g. ProactivePDC [14]) or message passing efficiency (e.g. Hyperion [15]). Many of these technologies have already been proven to be appropriate for rapid prototyping, involving traditional multiphysics and multi-scale models developed from different disciplines and organizations. What is crucially important for this project is the clean, simple and flexible programming model of mobile or static component systems, which is expected to facilitate the development of the 
overall envisioned system. Besides making necessary adjustments/extensions to such systems, our IT development work will also focus on transforming proven but largely monolithic legacy codes into a set of well integrated, interoperable components (via wrappers based on the Java Native Interface (JNI) [16]) that can be distributed over the network.

\section{Software Architecture of DDEMA Based on Data-Driveness Time Modalities}

The application domain for the validation of utility for DDEMA will be that a) of composite material structures in their linear and nonlinear behavioral regimes when they interact with surrounding fluids under multiphysics loading conditions primarily for material/structural design of supersonic platform and b) fire-material-environment interaction, monitoring, assessment and management system.

The two application scenarios considered can be characterized by the following data-driveness time modalities which influence the software architecture of DDEMA. We have identified the following two time modalities:

- Real time (unpredictable) data driveness where data, originating from sensor networks, effect systemic operational assessment of integrity and utility measures (i.e. fire effects on environment and structural/material health).

- Prior time (operator controllable) data driveness where massive data from automated experimentation are used to select, calibrate and construct behavioral models for materials/structures and fluids related to design, qualification and prediction scenarios (i.e. design of a nonlinear supersonic platform). This can also have a real time component as well especially if model selection is left to be determined by incoming sensor data both on laboratory and on-board platform (ships, submarines etc) situations.

Throughout, we will use these modalities to describe the two proposed DDEMA application architectures.

\subsection{Real-Time System}

In this system configuration, we assume that the on-board user has the many operational objectives some of which are:

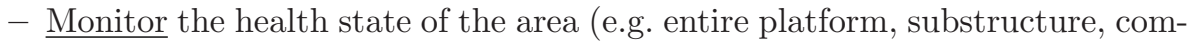
ponent) of interest by observing continuous field distributions guided by incoming sensor datastreams originating from a distributed sensor network that can be monitored from multiple points of entry.

- Simulate potential future states ("what-if" scenarios) of the system given certain hypothetical battle or situational escalation scenarios. This requires auto conversion of actual operating conditions to corresponding boundary/initial conditions, exploitation pre-computed field states to synthesize potential field evolutions quickly for "what-if" scenarios. 
- Based on input from the previous two items, the on-board users will be able to make decisions and implement them by activating actuation mechanisms. These require a dynamic analysis of simulated scenarios based on controllability of the systems, an impact analysis via a system that implements heuristic knowledge management techniques, and an archival capability for future re-usage and recording evaluative experiences of implemented decisions.

A high level schematic view of the so called real-time system architecture is depicted in Fig. 1, where the coarse grain functionality modules have been shown from a data association perspective only. This diagram should be implementable within the VPDE. Space limitations require that we do not expand on the definition of each one of the modules presented.

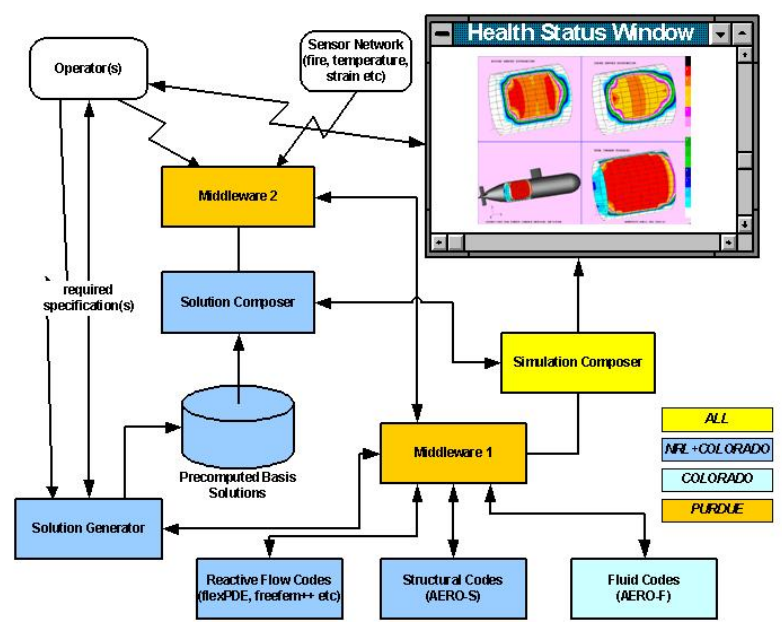

Fig. 1. A real-time system architecture.

\subsection{Prior-Time System}

In this system configuration, we assume that the laboratory or off-board user, who can be a structural designer or system qualifier who needs accurate systemic state behavior prediction, has similar operational objectives to that of the realtime modality. The only difference will be that the real-time component will not be present. The role of the incoming data will be played by the multidimensional material testing data-streams for identifying constitutive behavior of materials that may also guide model selection and implementation.

This requirements specification represents the structural system design and qualification utilization of the system. The high level schematic view of the so called prior-time system architecture is depicted by appropriate adjustments of the architecture depicted in Fig. 1. 


\section{The Design of Middleware and User Interface}

The plethora of available choices on computational infrastructure (hardware, software, networking), see Sec. 2, along with specific domain of application and user specific backgrounds and needs have introduced a large number of research issues associated with the computational implementation of any MPSE. The major of these issues are: (1) ability and optimization for computation over distributed resources, (2) ability for dynamic migrating component distribution, (3) adaptive modeling capability, (4) user dependent customization, (5) collaborative knowledge representation and interactivity, (6) dealing with heterogeneity of legacy and new code, and finally (7) ability to sustain component fault tolerance. To address several of the above issues, in the context of the data-driveness and the two time modalities scenarios considered, the following middleware design is proposed.

\subsection{DDEMA Middleware}

The design of the middleware is based on our experience from previous projects implementation, such as GasTurbinLab [1]. It is presented in the context of an agent-based middleware. New packages (e.g. Ptolemy II, [13]) are based on a combination of computational domain "director" and "actor" dual concepts. Actors can be thought as metamorphic agents with a visual incarnation in a GUI, and therefore include the concept of the agent and accordingly we promote their use in DDEMA.

Based on the issues, requirements, and data associations between functional abstractions as they have been described above, we have decided on proposing the following set of actors. A Director (or General Control Actor (GCA)), a Finite Element Tear and Interconnect Actor (FETIA), a Communication Actor (CA), Domain Actors (DA), Database Actor (DBA), and many I/O Actors (e.g. Display Actor, Printer Actor, Sensor Actor, User Actor), see Fig. 2.

Each Actor has specific tasks and interactions with some of the other Actors. However, we will not discuss here the individual responsibilities of each actor/agent due to the lack of space and we will allow the reader to infer some of this functionality through the associations depicted in Fig. 2

In many cases the actors to be developed will have to be constructed such as they encapsulate of existing codes like the AERO suite [5],[6] or their subcomponents.

\subsection{DDEMA Realization and User Interface/VPDE}

DDEMA's realization will be based on two main operational modes: The applications design and the application use modes.

In the application design mode the designer will utilize the VPDE for designing the actual application architecture in terms of data flow and message passing diagram. In particular, a visual representation of the linguistic components available for composition through their visual incarnations within the VPDE will be used. This is exactly why we plan to utilize an appropriately modified version 


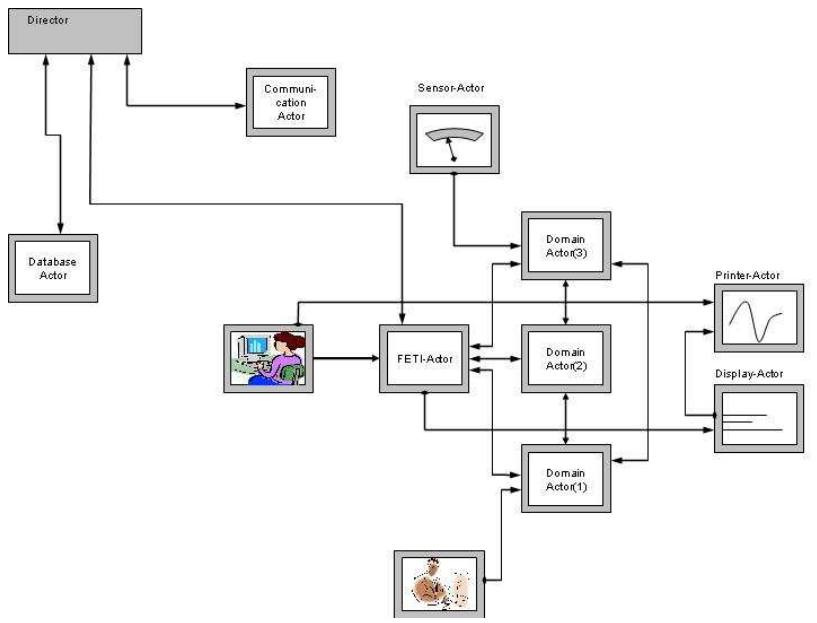

Fig. 2. Middleware design in Ptolemy's Vergil used as a VPDE.

of the "Vergil" visual editor paradigm provided by the Ptolemy II ([13]), system which already contains a well documented specification for creating new actors and directors. In this mode of usage DDEMA's GUI will be that of the VPDE based on Ptolemy-II.

In the application use mode, the user will take action to initiate the automatic generation of java source code or bytecode that implements the application functionality intended. Upon execution of the bytecode, the user will be presented with a stand alone custom made application that is the result of the previous mode to perform the activities associated with the corresponding usage requirements. During this phase secondary process will be spawned on the appropriate lightweight and heavyweight computational infrastructure available at hand. In order to address issues of parallelism, distribution, concurrency, security and process mobility, we will first attempt to introduce an intermediate layer between the actual mission functional and actor view layers, that provides a unified set of resources for this goal such as Inria's ProActive library [14]. In this case the GUI will be the one defined by the user in terms of the previous stage.

\section{Case Studies}

\subsection{Coupled Multiphysics of Continua: Material/Structural Design of Supersonic Platform}

Our experience with continuous multiphysics field theories [17], [18] suggests that the design process of a structure often requires the usage of a tool such as DDEMA for establishing optimal material characteristics like fiber-matrix properties, fiber orientation, and laminate layup properties, and the shape tailoring for an aircraft - or an aircraft component such as a fuselage, a wing, or a control 
surface - under high temperature and mechanical loading conditions inflicted from a supersonic mission requirement.

Intrinsic to the validity and confidence of any aero-structural simulation are the utilized continuous models of material behavior. It is exactly this area of where NRLs robotic testing capability along with historical exhaustive data pertaining to identification of material behavior, will be used as the springboard to launch an effort for automating model selection, implementation and verification methodologies. This will be done by considering all intrinsic and extrinsic, wanted and unwanted factors (uncontrolled biasing, noise, repeatability, history dependence etc) effecting data quality for the intended usage of model formation. Information theoretic, statistical and certainly deterministic techniques for model selection and/or generation in the context of continuous system identification and inverse approaches will be encapsulated in DDEMA. This will exercise the prior time data-driveness aspect of the system.

We have already demonstrated that it is possible to deal with most of these issues from a single physics rapid modeling perspective [8], [9]. Currently, the University of Colorado members of our team are part of a research consortium investigating the development of a supersonic aircraft shaping technology for reducing the initial shock pressure rise characterizing the ground signature. However, such a Quiet Supersonic Platform (QSP)requires a lightweight airframe, and will exhibit an aero-structural behavior. Its feasibility depends not only on the ability to design a shape that generates a low-boom ground signature, but most importantly on the ability to build a lightweight high-stiffness and damage tolerant structure that can withstand the expected aerodynamic and thermal loadings for long-range missions at a sustained rate. Therefore, the final design of a QSP will have to rely on a multidisciplinary aero-thermo-structural optimization. The DDEMA provides a unique opportunity to complement our current QSP effort. From the real time modality perspective, an on board version of a particular runtime version of DDEMA can be utilized to exploit sensor originated datastreams for helping responsible personnel to take decisions about restrictions or extensions of the platform operational envelope based on data driven simulation of potential outcomes of structural health metrics such as dissipated energy density. More details of how this expression of DDEMA can evolve is further expanded below for the case of our second application.

\subsection{Monitoring, Assessment, and Management of Fire-Material- Environment Interaction}

The ability to deal with the consequences of fire, extend over a wide range of application areas that have a direct effect on the survivability, ability to repair, maintainability, life extension and mission goals attainment of the environments and structural platforms effected by it. Some of these application areas can involve time critical situations that when they arise, demand decision-making support for an accurate monitoring capability supplemented by fire damage assessment and management and control countermeasures capabilities. A point in case are navy vessels build with a great variety of materials under extremely 
demanding threat conditions such as possible catastrophic events due to fuel tanks proximity, limited oxygen supply (i.e. submarines), fire byproducts toxicity, structural and atmospheric damage.

To demonstrate the value of the core ideas behind the development of DDEMA, we plan develop a proof of concept instantiation of DDEMA within the context of managing all required activities of an accidental fire induced crisis scenario. More specifically, this application of DDEMA should be able to provide assistance to the users by employing the following features: (1) Ability to receive real time datastreams from multiple redundant distributed sensor networks that allow capturing of multimodal point or continuous distributions of various field quantities such as area/volume of live flames, temperature, chemical byproducts concentrations etc; (2)ability for multi-point-of-entry monitoring where this can be accomplished in multiple locations synchronously or asynchronously; (3) copresentation of reactive flow and reactive phase transformation simulation capability with multiphysics fluid-structure interaction simulation capability that allows "what-if" prediction exploration in order to be able to evaluate validity of decisions and alternative countermeasures; (4) a decision support subsystem, in order to combine sensor inputs, simulation results analysis and user choices based on experience and knowledge and form a course of action countermeasures that can also be simulated; (5) an interface to a control system of an existing countermeasure distributed actuation network, in order to implement the decided management strategy.

A system with these capabilities allows for portability and wide applicability.

Acknowledgement. The authors acknowledge the support by the National Science Foundation under grants EIA-0205663 and EIA-0203958.

\section{References}

1. Fleeter, S., Houstis, E.N., Rice, J.R., Zhou, C., Catlin, A.: GasTurbnLab: A Problem Solving Environment for Simulating Gas Turbines. Proc. 16th IMACS World Congress, (2000) No 104-5.

2. Houstis, E.N., Catlin, A.C., Tsompanopoulou, P., Gottfried, D., Balakrishnan, G., $\mathrm{Su}, \mathrm{K}$. , Rice, J.R.: GasTurbnLab: A Multidisciplinary Problem Solving Environment for Gas Turbine Engine Design on a Network of Non-Homogeneous Machines. To appear in the Proceedings of 15th Toyota Conference, October 2001 and special issue on the J. of Computational Engineering and Mathematics.

3. Rice, J.R., Tsompanopoulou, P., Vavalis, E.A.: Interface Relaxation Methods for Elliptic Differential Equations. Applied Numerical Mathematics 32 (1999) 219-245.

4. Tsompanopoulou, P.: Collaborative PDEs Solvers: Theory and Practice. PhD thesis, Mathematics Department, University of Crete, Greece (2000).

5. Farhat, C., Lesoinne, M.: Two Efficient Staggered Procedures for the Serial and Parallel Solution of Three-Dimensional Nonlinear Transient Aeroelastic Problems. Computer Methods in Applied Mechanics and Engineering, 182, (2000) 499-516.

6. Farhat, C., Lesoinne, M., Stern, P., Lanteri, S.: High Performance Solution of ThreeDimensional Nonlinear Aeroelastic Problems Via Parallel Partitioned Algorithms: Methodology and Preliminary Results. Advances in Engineering Software, 28 (1997) 43-61. 
7. Tran, H., Farhat, C.: An Integrated Platform for the Simulation of Fluid-StructureThermal Interaction Problems, AIAA J. (in press.)

8. Michopoulos, J., Badaliance, R., Chwastyk, T., Gause, L., Mast, P., Farhat C., Lessoine, M.: Coupled Multiphysics Simulation of Composite Material Softening in a Virtual Wind Tunnel Environment, invited paper in Proceedings of Sixth U.S. National Congress on Computational Mechanics, U.S. Association for Computational Mechanics, Dearborn MI, (2001) pp. 521.

9. Michopoulos, J., Farhat, C., Lesoinne, M., Mast, P., Badaliance, R., Chwastyk, T., Gause, L.: Material Softening Issues in a Multiphysics Virtual Wind Tunnel Environment, AIAA Paper 2002-1095, 40th Aerospace Sciences Meeting and Exhibit, Reno, Nevada, (2002).

10. The Grasshopper Agent Platform, IKV++ GmbH, Kurfurstendamm 173-174, D10707 Berlin, Germany. http://www.ikv.de

11. Java-RMI-IIOP: http://java.sun.com/products/rmi-iiop/

12. Parker, L., Christensen, S.M.: MathTensor: A System for Doing Tensor Analysis by Computer, by Addison-Wesley, (1994)

13. Davis II, J., Hylands, C., Kienhuis, B., Lee, E.A., Liu, J., Liu, X., Muliadi, L., Neuendorffer, S., Tsay, J., Vogel, B., Xiong, Y.: Heterogeneous Concurrent Modeling and Design in Java, Memorandum UCB/ERL M01/12, EECS, University of California, Berkeley, CA USA 94720 March 15, 2001, http://ptolemy.eecs.berkeley.edu/ptolemyII/

14. Caromel, D., Klauser, W., Vayssiere, J.: Towards Seamless Computing and Metacomputing in Java, pp. 1043-1061 in Concurrency Practice and Experience, September-November 1998, 10(11-13). Editor Geoffrey C. Fox, Published by Wiley \& Sons, Ltd., http://www-sop.inria.fr/oasis/proactive/

15. Antoniu, G.L., Bouge, l., Hatcher, P., MacBeth, M., McGuigan, K., Namyst, R.: Compiling multithreaded Java bytecode for distributed execution. In Euro-Par 2000: Parallel Processing. Lecture Notes in Comp. Science, Vol. 1900. Springer-Verlag, Munchen, Germany, (2000) 1039-1052.

16. Java Native Interface Specification. http://web2.java.sun.com/products/jdk/1.1/docs/guide/jni.

17. Sih, G.C., Michopoulos, J.G., Chou S.C.: Hygrothermoelasticity. Martinus Nijhoff Publishers (now Kluwer Academic) (1986).

18. Michopoulos, J.G., Sih, G.C.: Coupled Theory of Temperature Moisture Deformation and Electromagnetic Fields. Institute of Fracture and Solid Mechanics report IFSM-84-123, Lehigh University, (1984). 